\title{
Development and Characterisation of Nonwoven Fabrics for Apparel Applications
}

\author{
Cheema SM*, Shah TH, Anand SC and Soin N
}

Institute of Materials Research and Innovation (IMRI), University of Bolton, Deane Road, Bolton BL3 5AB, UK

\begin{abstract}
The cost of making apparel fabrics for garment manufacturing is very high because of their conventional manufacturing processes and new methods/processes are being constantly developed for making fabrics by unconventional methods. With the advancements in technology and the availability of the innovative fibre, durable nonwoven fabrics by using the hydroentanglement process that can compete with the woven fabrics in terms of their aesthetic and tensile properties are being developed. In the work reported here, the hydroentangled nonwoven fabrics were developed through a hybrid nonwoven manufacturing processes by using fibrillated Tencel ${ }^{\circledR}$ and bi-component (sheath/core) polyethylene/polyester (PE/PET) fibres, in which the initial nonwoven fabrics were prepared by the needle-punching method followed by hydroentanglement process carried out at optimal pressures of 50 to 250 bars. The prepared fabrics were characterised according to the British Standards (BS 3356:1990, BS 9237:1995, BS 13934-1:1999) and the attained results were compared with those for a standard plain-weave cotton, polyester woven fabric and commercially available nonwoven fabric $\left(\right.$ Evolon $\left.^{\circledR}\right)$. The developed hydroentangled fabrics showed better drape properties owing to their flexural rigidity of $252 \mathrm{mg} . \mathrm{cm}$ in the $\mathrm{MD}$, while the corresponding commercial hydroentangled fabric displayed a value of $1340 \mathrm{mg} . \mathrm{cm}$ in the MD. Tensile strength of the developed hydroentangled fabrics showed an approximately $200 \%$ increase than the commercial hydroentangled fabrics. Similarly, the developed hydroentangled fabrics showed higher properties in term of air permeability, such as the developed hydroentangled fabric exhibited $448 \mathrm{~mm} / \mathrm{sec}$ and Evolon fabric exhibited $69 \mathrm{~mm} / \mathrm{sec}$ at $100 \mathrm{~Pa}$ pressure. Thus for apparel fabrics, the work combining the existing methods of nonwoven production, provides additional benefits in terms of cost, time and also helps in reducing the carbon footprint for the apparel fabric manufacture.
\end{abstract}

Keywords: Hydroentanglement; Nonwoven apparel; Durable nonwovens; Tencel; Evolon

\section{Introduction}

Traditionally, it is being assumed that garments are made through woven and knitted fabrics. Because of their acceptable aesthetical and mechanical properties, these conventional methods of making fabrics for garments captured a big market. From the literature review, it was found that there was very limited penetration of nonwoven fabrics in outerwear owing to their inherent limitations in term of strength, appearance and workability. In 1960, for the first time nonwoven fabric was developed as an outer wear, but could not succeed came as outer fabric but could not success because of its limitations. Nonwoven fabrics are thus being used as supporting materials in garment manufacturing industry, such as garment lining, insulation, and fusing etc. Recently, there has been a great deal of interest in the area of research and innovation activities in nonwoven fabrics for apparel applications, such as Evolon and Miratech [1]. Traditionally, it was assumed that nonwoven fabrics can only perform in wipes, scaffolds, geo textiles, filters and disposable articles and only 1 percent of nonwoven fabrics are utilised in apparel sector $[2,3]$. Now, because of the new advancements in the nonwoven technologies, especially in the hydroentanglement process, the applications of hydroentangled nonwoven fabrics have diversified into many fields and apparel fabrics application is one of them. The key area of research is to develop the nonwoven fabrics that can withstand the external forces in the use and provide comfort to the wearer in terms of softness, moisture management, appearance and also exhibit enough strength that can withstand the laundry process etc. [4].

Nonwoven fabrics are made directly from the fibres without making any yarn and without the use of weaving or knitting processes. A nonwoven fabric is defined by INDA as follows: "Sheet or web structure bonded together by entangling fibres or filament, by various mechanical, thermal and/or chemical processes. These are made directly from the separate fibres or from molten plastic or plastic film". The unique advantage of the nonwoven fabric manufacture is that it is a continuously linked process, in which at the first stage raw materials (fibres) are converted into webs via the carding process and at the second stage these fibrous webs are bonded into finished products. The first nonwoven apparel fabric was the disposable paper clothing that was launched in early 1966 by an American company Scott Paper, however, the fabric could not capture any significant part of the apparel market because of its ill fit and uncomfortable nature. In the same era, Mars Manufacturing Company invented different types of nonwoven dresses that were used as evening wear and wedding gowns. However, these all-paper-clothing were short-lived and became obsolete in 1968 [5]. The breakthrough in the nonwoven apparel fabrics came in 1970, when DuPont invented the hydroentanglement technique for the nonwoven fabrics, known as spunlace technology [5]. This new technique gave a competitive edge to nonwovens, as the nonwoven fabrics produced by the hydroentanglement process possessed much superior aesthetical properties than the conventional nonwovens. The company also developed the foam bonded spunlaced fabric (polyester spunlaced fabric), wherein $30 \%$ soft acrylic latex was used to strengthen the spunlaced fabric that withstood five laundering cycles [6].

*Corresponding author: Cheema SM, Institute of Materials Research and Innovation (IMRI), University of Bolton, Deane Road, Bolton BL3 5AB, UK, Tel: 00447735564985; E-mail: chscheema@gmail.com

Received May 05, 2018; Accepted May 14, 2018; Published May 23, 2018

Citation: Cheema SM, Shah TH, Anand SC, Soin N (2018) Development and Characterisation of Nonwoven Fabrics for Apparel Applications. J Textile Sci Eng 8: 359. doi: 10.4172/2165-8064.1000359

Copyright: (c) 2018 Cheema SM, et al. This is an open-access article distributed under the terms of the Creative Commons Attribution License, which permits unrestricted use, distribution, and reproduction in any medium, provided the original author and source are credited. 
In this study, functional hydroentangled fabrics have been developed through a hybrid process of needling and hydroentanglement, by utilising innovative Tencel and bi-component sheath/core (PE/PET) staple fibres. The resultant fabrics, when compared against the industry standard commercially available Evolon ${ }^{\infty}$ and woven plain-weave cotton/polyester fabric exhibited acceptable test values related to the aesthetical and mechanical properties of the garments such as flexural rigidity, air permeability and tensile strength etc. The resultant fabrics can be used in different applications such as in the hospital for patients uniforms. Owing to its comfortability, it can be used in the processing industry as uniforms such as in the meat industry, printing industry and in the chemical industry. Further research work is being carried out in order to enhance the quality of the fabrics especially in relation to laundering and washing.

\section{Experimental Section}

\section{Materials}

The fabrics were produced in collaboration with the Nonwovens Research Institute, University of Leeds by utilising two different types of fibres: Tencel $^{\varpi}$ and bi-component sheath/core (PE/PET) staple fibres. Tencel ${ }^{\triangleright}$ fibres were $1.4 \mathrm{dtex}, 38 \mathrm{~mm}$ in length with a smooth surface, while the PE/PET bi-component fibres were 2.2 dtex and 40 $\mathrm{mm}$ in length with a crimped surface. The required amount of each fibre was separately weighed and initially hand blended in preparation of the next process. These hand-mixed fibres were manually opened into small tufts and further mixed in order to obtain a smooth web. After manually opening the fibres, the fibres were then carded through the pilot carding machine and a parallel-laid web produced was used in the next process. Before going into the hydroentanglement process, the carded web was lightly needled by using $8 \mathrm{~mm}$ penetration and 100 strokes per min, after which the needled substrate was rolled and packed for the next process of hydroentanglement.

\section{Hydroentanglement process}

The needled fibrous webs were uniformly hydroentangled by using pilot hydroentanglement system, as illustrated in Figure 1. Before going under the high pressure area of the hydroentanglement process, the fibrous web was pre-wetted to minimise the dispersion ratio of the fibres in high pressure zone. After pre-wetting, the material is passed through the high water pressure jet head for bonding of the fibres as shown in Figure 1. The Fabrics were prepared at varying hydroentanglement pressures of 100 and 125 bars with two passes, at the line speed of $3 \mathrm{~m} / \mathrm{min}$. The orifice of jet strip was $150 \mu \mathrm{m}$ and the density of the jet orifice was $5.56 / \mathrm{cm}$. All the fabrics were manufactured from the same web with $120 \pm 5$ gm- 2 nominal weight. It was observed that for these specific blends, at lower pressures (50-75 bars), the fibres

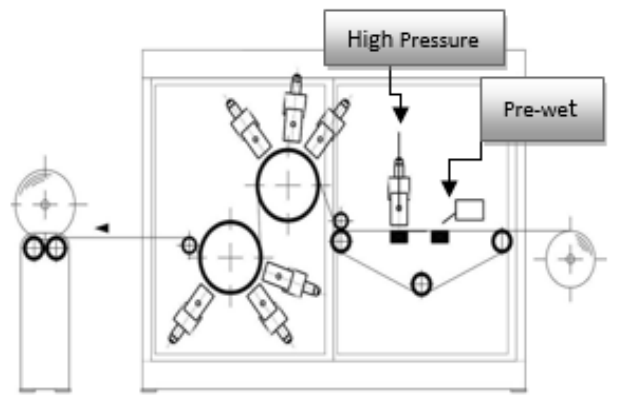

Figure 1: Action view of hydro entanglement process. were not consolidated to the required level, which negatively affected the mechanical properties by reducing the tensile strength of the obtained fabric. On the other hand, at higher pressures (150-250 bars), the fibres were easily dispersed and consequently very limited number of fibres per unit area of the fabric was observed, which reduced the GSM as well as the tensile properties to below the acceptable values. The two hydroentangled nonwoven fabrics denoted as fabric 1 and fabric 2, were prepared at 100 and 125 bars, respectively, at the same constituent levels of 70\% Tencel and 30\% PE/PET bi-component fibres. The properties of developed fabrics are shown in Table 1.

\section{Mechanical and aesthetical testing}

The prepared fabrics were tested according to the British Standards (BS 3356: 1990, BS 9073-6: 2003, BS 13934-1: 1999) in order to determine their mechanical and aesthetical properties. For flexural rigidity, Shirley flexometer apparatus was used, where a $25 \pm 1 \mathrm{~mm}$ $\mathrm{x} 100 \pm 1 \mathrm{~mm}$ strips were cut and the flexural rigidity values were determined according to the standard procedures. The tensile tests were performed by the Instron apparatus according to the appropriate standard methods. The cross-head speed was $200 \mathrm{~mm} \pm 1 / \mathrm{min}$ and the fabric size was $200 \pm 1 \times 50 \pm 1 \mathrm{~mm}$. Three specimens of each fabric were tested. The wicking test was performed by strip test method and absorption test was carried out by dipping $(10 \mathrm{~cm} \times 10 \mathrm{~cm})$ fabric specimens into water for $20 \mathrm{~min}$ at $20^{\circ} \mathrm{C}$ and $65 \pm 2$ relative humidity.

The results obtained were then compared with the commercially available nonwoven fabric "Evolon" and with the reference fabric of a plain weave of woven fabric to ascertain the suitability of our developed fabrics for use as apparel fabrics.

\section{Results and Discussion}

\section{Dimensional properties}

The results presented in Figure 2 show that fabrics 1 and 2, produced at 100 and 125 bars hydro pressure had thickness values of $0.99 \pm 0.05 \mathrm{~mm}$ and $0.88 \pm 0.05 \mathrm{~mm}$, respectively. This indicates that when the hydroentanglement pressure was increased, a small reduction in the fabric thickness was observed due to a higher compaction and alignment of the fibres, which is expected to influence the tensile properties and bending rigidity of the produced fabric. In a study by Zheng et al., it was shown that by increasing the specific energy (hydro pressure), the fabric area density decreased due to the fabric stretching caused by the impact of the water jets.

Moreover, it was observed that both the fabrics exhibited almost very similar bulk density values, which is an indication that the number of fibres per unit area in these fabrics were similar to each other. When compared to the values for the commercial nonwoven fabric and a typical woven fabric, these comparative results clearly show that the dimensional properties such as thicknesses of the hydroentangled nonwoven fabrics ( 1 and 2) were higher, because fabric 1 exhibited $0.99 \pm 0.05 \mathrm{~mm}$ and fabric 2 exhibited $0.88 \pm 0.05 \mathrm{~mm}$ thickness and Evolon and woven fabrics showed 0.43 and $0.50 \mathrm{~mm}$ thickness values respectively. The bulk densities of both fabrics were lower than Evolon ${ }^{\circ}$ and woven fabrics. Woven and Evolon fabrics' higher bulk density values were because of their compact structures as shown in Table 2.

\section{Flexural rigidity}

The flexural rigidity of the fabrics is a pivotal property for apparel applications. The fabric stiffness is related to its inherent properties, such as fibre material and the structure itself of the fabric [7]. The 


\begin{tabular}{|c|c|c|c|c|c|}
\hline Fabric Number & Hydroentanglement Pressure (bars) & Contents & Area Density $\left(\mathrm{gm}^{-2}\right)$ & Thickness (mm) & Bulk Density gc $\left(\mathrm{m}^{-3}\right)$ \\
\hline Fabric 1 & 100 & Tencel $(70 \%)$ and Sheath core PE/PET (30\%) & $150 \pm 2.5$ & $0.99 \pm 0.05$ & $0.15 \pm 0.01$ \\
\hline Fabric 2 & 125 & Tencel $(70 \%)$ and Sheath core PE/PET (30\%) & $145 \pm 2.5$ & $0.88 \pm 0.05$ & $0.17 \pm 0.01$ \\
\hline
\end{tabular}

Table 1: Details and characteristics of the hydroentangled nonwoven fabrics prepared in this study.

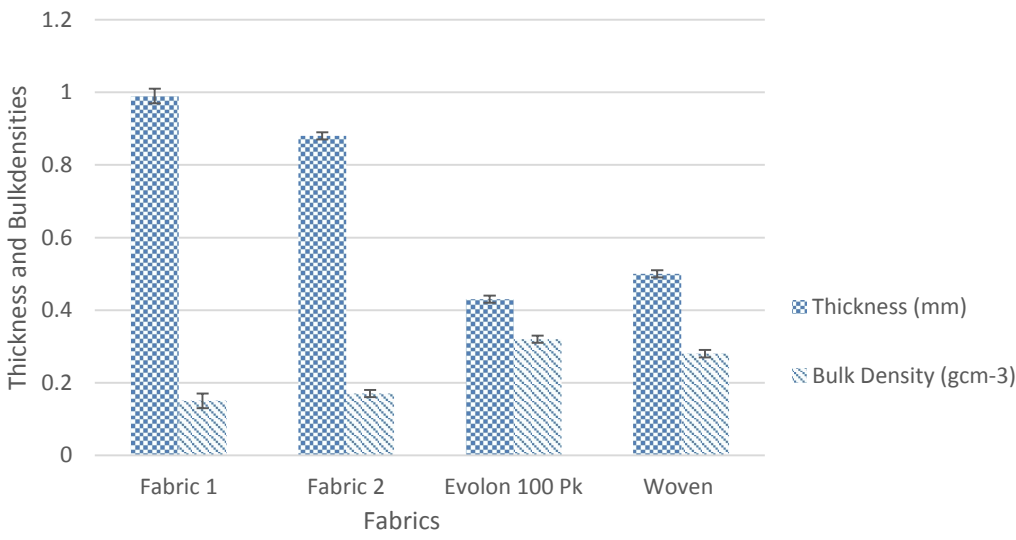

Figure 2: Thickness and bulk density of developed fabrics 1 (100 bars) and 2 (125 bars)

\begin{tabular}{|c|c|c|c|c|c|}
\hline Fabrics & Processes & Contents & Area Density $\left(\mathrm{gm}^{-2}\right)$ & Thickness (mm) & Bulk Density $\left(\mathrm{gcm}^{-3}\right)$ \\
\hline Evolon $^{\circledR}$ & Hydroentanglement & PET and PA & 140 & 0.43 & 0.32 \\
\hline Woven & Plain weave & Cotton and PET & 144 & 0.50 & 0.29 \\
\hline
\end{tabular}

Table 2: Details of reference fabrics.

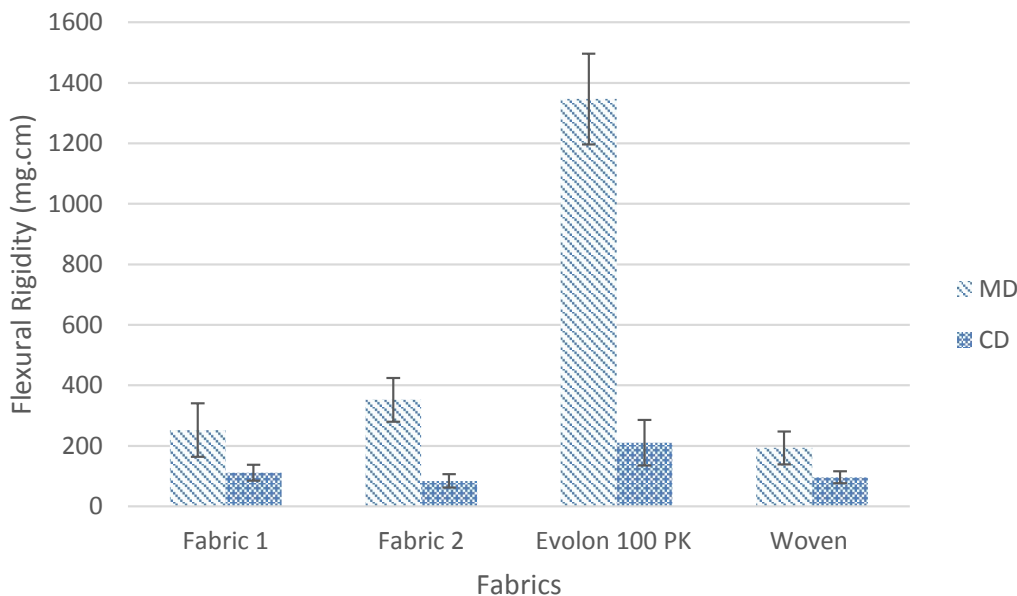

Figure 3: Flexural rigidity comparison of developed fabrics 1 and 2 with Evolon 100PK and Woven fabric.

flexural rigidity results in the machine (MD) and cross (CD) directions for the fabrics produced during this research are presented in Figure 3. It was observed that the flexural rigidity values for the nonwoven fabrics prepared in this study were much lower than the commercial nonwoven fabrics (Evolon ${ }^{\circ}$ ) in both the MD and CD. Furthermore, these values were very similar to those observed for the woven fabric (Figure 4). These results clearly show that the flexural behaviour of fabrics 1 and 2 was very similar to that of the woven fabric and it can be further enhanced through the finishing processes.

The bending rigidity of the fabrics depends on the movement of the fibres within the fabric structure. It seems that the Evolon fabrics exhibit higher bending rigidity as the fibres are highly intertwined with its neighbouring fibres due to the nature of the spun-laid process and the inherent fine filaments of the bi-component PA6/PET "island in the sea" filament structure. Additionally, due to the high hydroentanglement pressure, the fine filaments form a tight structure with little or no space for fibre movement to occur within the fabric. On the other hand, the prepared fabrics showed lower flexural rigidity as compared to the commercial nonwoven fabric due to the more open structure of these experimental nonwoven fabrics. In their study, Smith et al. have reported that if the fibres are able to act independently in the fabric structure, a reduction in the flexural rigidity of the fabric is observed [8]. Hence, it is evident that bending rigidity does not depend on the thickness of the fabric and rather is directly proportional to the movement of the fibres within the structure of the fabric. Evolon ${ }^{\circ}$ exhibited higher flexural rigidity in both the machine and cross 
directions, even though it had a lower thickness value. On the other hand, the developed hydroentangled nonwoven fabrics exhibited lower flexural rigidity in the machine and cross directions, as compared with the Evolon, while showing higher thickness values than the Evolon ${ }^{\circ}$ fabric. Woven fabric had similar thickness as compared with the Evolon ${ }^{\oplus}$ but exhibited much lower values' of flexural rigidity in the machine and cross direction. Anastasija et al. found in their studies that the fabric structure has a more significant effect on the flexural rigidity of the fabric [9]. Emin et al. too have endorsed this principle that the fabric parameters affect the stiffness of the fabric [10].

Figure 4 represents the microscopic images of the three different types of fabrics studied during the course of the work. These images show that the nonwoven fabrics developed in this study and the woven fabric have similar open structures and the fibres appear to be better aligned than the commercially available Evolon, wherein the fibres appear to be randomly placed. Komori found that the arrangement of the fibres in the fabric structure has a major influence on the mechanical properties of the fabric [11]. Thus, the experimental nonwoven and woven fabrics exhibit similar flexural behaviour and have significantly lower flexural rigidity values as compared to the commercial nonwoven fabric. In the developed hydroentangled fabrics, the fibres are largely aligned in a single direction and the open spaces between the fibres can lead to the lowering of the flexural rigidity. Similar results have been obtained by Bahari et al. and Smith et al. wherein it was observed that the fibre's independent movement within the fabric structure led to a lower fabric flexural rigidity $[8,11]$.

\section{Absorption and wicking tests}

The moisture transportation in a fabric determines its cooling effect and thus provides comfort to the wearer. The wear comfort of clothing is continually gaining importance owing to the consumer demand and therefore considerable efforts have been made to study the wicking characteristics of apparel fabrics [12,13]. During exercise or working conditions, the human body produces sweat and if it does not evaporate from the skin to the atmosphere, because of the clothing, then the wearer feels uncomfortable and this may give rise to a loss in working efficiency of the wearer. Therefore moisture management by the fabric is very important in order to optimise the wearer's comfort. The fibre types (natural or synthetic), blending ratio of fibres, the fabric structure and fabric characteristics (densities of yarns, thickness) etc. are the parameters that affect the moisture management and the thermal properties of the fabrics [14]

The prepared nonwoven and woven fabrics were characterised for their absorption and wicking properties and the results are presented in Table 3 and illustrated in Figure 5. The results show that the experimental fabrics 1 and 2 exhibit better absorption and wicking behaviour than Evolon 100 PK and the woven fabric. The absorption value of fabric 1 was $~ 78 \%$ higher than the woven fabric and about 90\% higher than Evolon 100 PK. Similarly, fabric 2 exhibited 70\% and $86 \%$ higher absorption values than woven and Evolon fabrics, respectively. This enhanced absorption is mainly due to the fact that the experimental nonwoven fabrics have a more porous structure and the

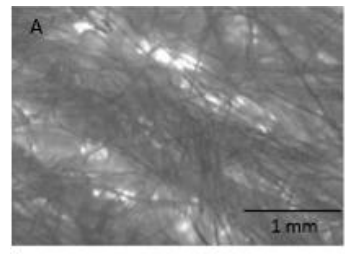

Developed nonwoven sample

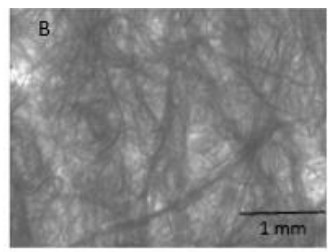

Evolone

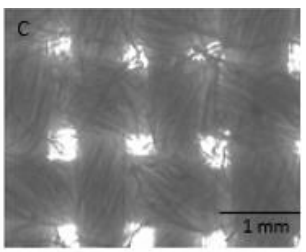

Woven fabric sample

Figure 4: Microscopic Views of (a) developed hydroentangled, (b) Evolon ${ }^{\circledR}$ and (c) woven

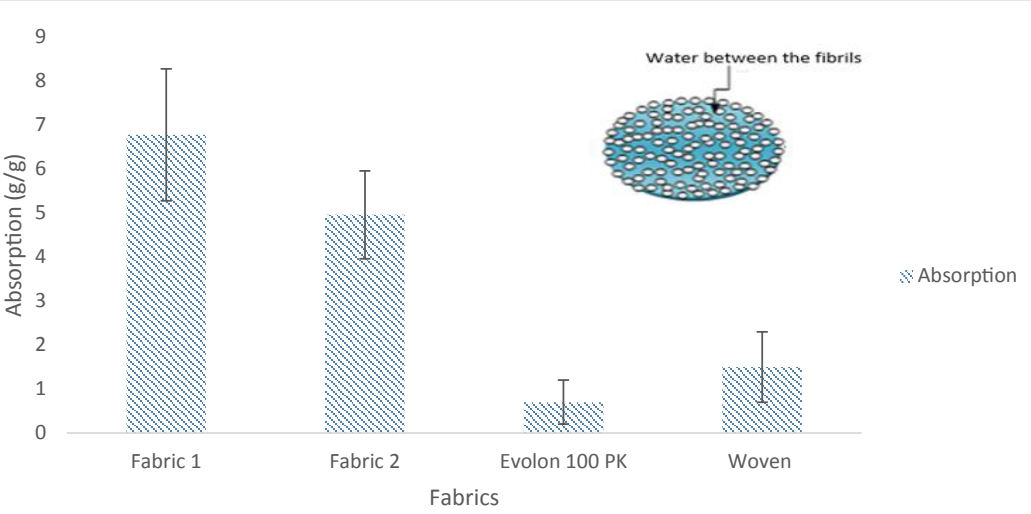

Figure 5: Absorption properties of nonwoven fabrics.

\begin{tabular}{|c|c|c|c|}
\hline Fabrics & Area Density gm $^{-2}$ & Absorption gg $^{-1}$ & Wicking (g.cm) \\
\hline Fabric 1 & & & MD \\
\hline Fabric 2 & 150 & 6.78 & 9.61 \\
\hline Evolon® ${ }^{-2}$ & 145 & 4.96 & 12.44 \\
\hline Woven & 140 & 0.7 & 1.4 \\
\hline
\end{tabular}

Table 3: Absorption and wicking properties of nonwoven fabrics. 
fibres are well oriented within the fabric structure. Moreover, owing to the Tencel fibres' hygroscopic nature, it absorbs the water molecules readily, thus providing higher absorption values. Furthermore, the Tencel $^{\oplus}$ fibres used have a higher wet and dry strength than the other cellulosic fibres, viscose fibres show $22-26 \mathrm{cN} /$ tex tenacity in dry state and $10-15 \mathrm{cN} /$ tex in wet state and on the other hand Tencel fibres show $34-38 \mathrm{cN} /$ tex and $38-42 \mathrm{cN} /$ tex in dry and wet states respectively and have higher moisture regain properties than the polyamide and polyester fibres used in the commercial nonwoven fabric (Evolon). Additionally, the fibril structure of Tencel fibres provides superior capillary action within the fabric structure [15]. Woven fabric displayed restricted absorption properties owing to its fabric structure, wherein, due to the high number of crimps per unit area, along with the highly twisted yarns, did not allow the fibres to act as capillaries, unlike the exhibited properties of the nonwoven fabrics.

The results presented in Table 3 also show that the wicking values obtained for fabrics 1 and 2 were considerably higher than the woven fabric and Evolon 100 PK nonwoven fabrics, both in the MD and CD. The wicking properties mainly depend on the fibre fineness and the fabric structure. Fabric 1 and 2 showed 9.61 and 12.44 g.cm wicking values in MD where as Evolon and woven exhibited wicking values of 1.4 and 1.4 g.cm in MD. The higher wicking values of the developed fabrics were because of the Tencel fibres consist of countless hydrophilic and crystalline nano fibrils which act as tubes and absorb the water through the capillary action between the fibrils [16]. On the other hand, Evolon, composed of polyester and polyamide fibres, show lower absorption, as the synthetic fibres like polyester are hydrophobic in nature. The fibre orientation also contributes in the absorption values of the fabric,
Miller has studied the pore size effects on the capillary behaviour of the material. For the developed hydroentangled fabrics, the smaller pore size along with the higher capillary action from Tencel fibres led to the enhanced wicking properties, as compared to the other fabrics.

\section{Tensile properties}

The tensile properties of fabrics 1 and 2 in MD and CD are shown in Figure $6 \mathrm{a}$ and $6 \mathrm{~b}$, respectively. The results show that the tensile strength of fabrics 1 and 2 are higher than the Evolon 100 PK and woven fabric in MD (Figure 6a). It also appears that the tensile behaviour of the fabrics 1 and 2 is significantly different from both the woven and the commercial nonwoven fabrics. As mentioned earlier, the developed fabrics were prepared at 100 and 125 bars, respectively. In their study Cannoly et al. have observed that an increase in the hydroentanglement pressure results in an increase in the tensile strength due to the higher degree of entanglement of the fibres [16]. The tensile strength also depends on other factors, such as the fibre and web properties. As discussed previously by Ghassemieh [17], to prepare a hydroentangled fabric, a sufficient amount of energy is essential to get an optimal product with suitable dispersion and sufficient tensile strength, which itself depends on the web making parameters and fibre properties such as modulus, stiffness, length and friction between the fibres. Mao found in his research that less stiff fibres consumed less energy for attaining the maximum fibre entanglement within the fabric structure [18]. Moreover, because of the staple fibres in the developed fabrics, during hydroentanglement process, more fibres were twisted around with surrounding fibres, because of the rebound of the water after hitting the plate underneath the web, resulted in intensive entangling behaviour of the fibres that caused the higher tensile strength of the developed
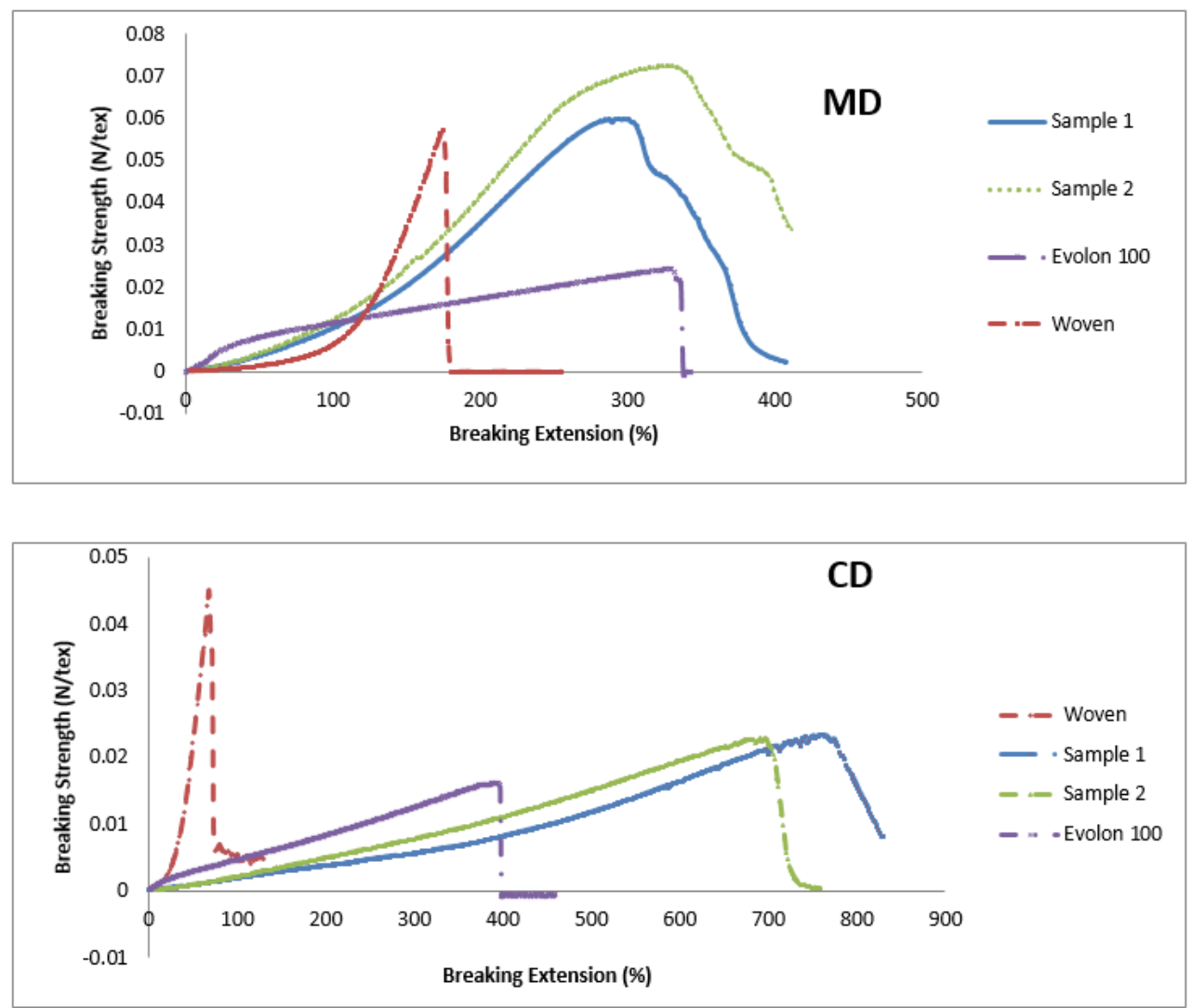

Figure 6: Tensile tests of different nonwoven fabrics in MD and CD. 
fabrics as compared with other fabrics [19-21]. The experimental nonwoven fabrics have breaking extension values that are similar to the Evolon fabrics; however their failure mechanism is quite different as these fabrics tend to yield before they fail. Due to its rigid structure, the woven fabric exhibited higher modulus and lower breaking extension values as compared to the nonwoven fabrics [22].

The tensile properties of the various fabrics in CD are illustrated in Figure $6 \mathrm{~b}$. The results show that the ultimate strength values of these experimental nonwovens are slightly lower than the woven fabric but higher than Evolon 100 PK. The breaking extension values of all the nonwovens are in excess of $80 \%$ and these are considerably higher than the woven fabric. The Evolon fabric is composed of continuous filaments of bi-component Island in the sea that can impart higher strength to the fabric, whereas the woven fabric is composed of twisted yarns with a compact weave that give additional strength to the fabric. On the other hand, fabrics 1 and 2 are composed of fine staple fibres and the fibres were aligned in machine direction so because of less number of fibres in the cross direction region, the developed fabric exhibited lower tensile strength in CD. Evolon fabric is also based on synthetic fibres and is produced by using split able bi-component fibres which are finer in diameter than those used for preparation of the experimental fabrics and are therefore able to entangle more intensely in the fabric structure and also the filaments were in different directions that caused higher tensile strength in $\mathrm{CD}$ than the developed fabrics as shown in Figure 7.

\section{Conclusions}

In this study, an attempt has been made to develop nonwoven fabrics that are suitable for apparel applications. A hybrid nonwoven approach has been adopted where the fibres are first converted into a parallellaid web followed by needle punching of the web. The needle punched fabric is then subjected to the hydroentanglement process at optimal values of 100 and 125 bars. The results demonstrate that the nonwoven fabrics produced in our study have superior moisture management properties such as developed fabric gave $6.78 \mathrm{~g} / \mathrm{g}$ values of absorption
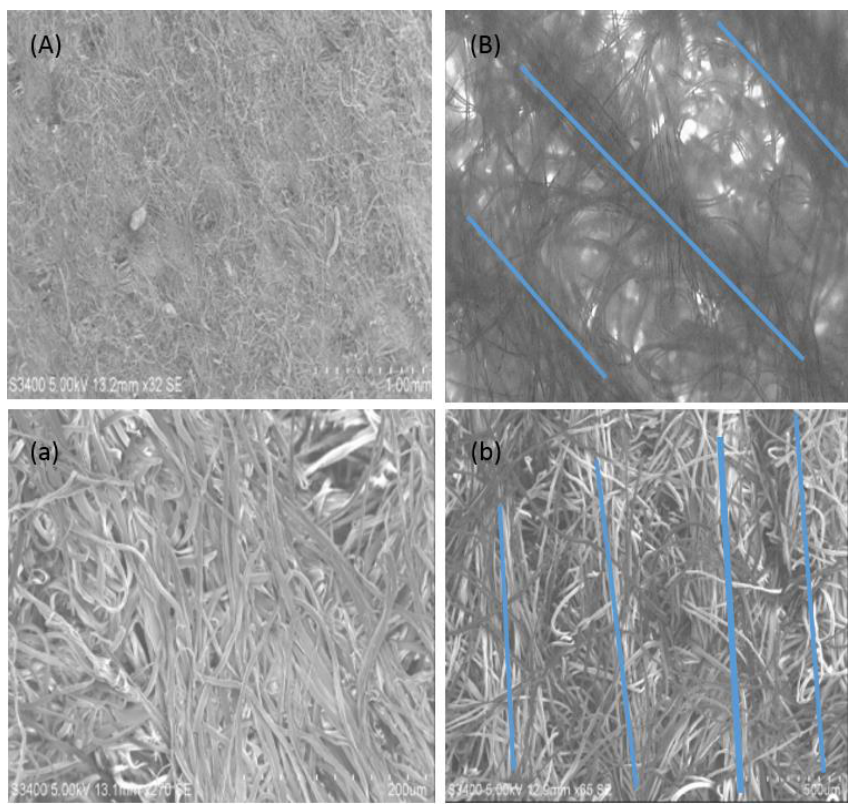

Figure 7: Optical and SEM views of Evolon 100 PK (A,a) and developed hydroentangled fabric $(B, b)$ and Evolon and woven gave 0.7 and $1.5 \mathrm{~g} / \mathrm{g}$, respectively. The bending flexural rigidity characteristics of the developed fabrics were also lower than the Evolon fabrics, wherein the developed fabrics exhibited 252 mg.cm in MD while the Evolon fabrics showed a value of $1347 \mathrm{mg} . \mathrm{cm}$ in MD. The tensile properties of the experimental nonwoven fabrics are higher than the commercially available nonwoven fabrics, especially the breaking extension values. The developed fabric exhibited a value of $0.07 \mathrm{~N} /$ tex in MD and Evolon exhibited value of $0.02 \mathrm{~N} /$ tex in MD. The tensile strength of the woven fabric is somewhat higher in CD than the nonwoven fabrics, but the bending rigidity values are very similar to the experimental nonwovens. The absorption and wicking properties of the developed fabrics were higher than the Evolon and woven fabric in both the machine and cross directions because of the hygroscopic and fibril structure of the Tencel fibres. The developed fabric exhibited an absorption value of $6.78 \mathrm{~g} / \mathrm{g}$ and Evolon fabric exhibited $0.7 \mathrm{~g} / \mathrm{g}$ absorption value. This prove that the developed fabrics quickly absorb the sweat from the body and because of their strong wicking action, the sweat disperses in the fabric structure that assists in the evaporation into the environment. The wicking values of developed fabric fabrics were 9.61 and 12.44 g.cm and the wicking values of Evolon and woven fabrics were 1.4 and 1.4 g.cm respectively. It is strongly believed that with further optimisation of the hybrid process parameters and the fabric finishing techniques, nonwoven textile structures can be developed that are suitable for many apparel applications, including patients' uniforms, uniforms for processing industries, outer wear etc.

\section{Acknowledgements}

The authors wish to thank Dr S. Rathore at NRI, University of Leeds, for his help with the processing of experimental fabrics.

\section{References}

1. www.evolon.com.

2. Luki S, Jovani $P$ (2004) Structural analysis of abrasive composite material with nonwoven textile matrix. Material Letters 58: 439-443.

3. Lee HJ, Cassill N (2006) Analysis of World Nonwoven Market. Journal of Textile and Apparel, Technology and Management 5: 10-18.

4. Vaibhav K, Webster L, Govekar A (2012) Nonwoven in fashion appare applications. International Journal of Fibre and Textile Research 2: 12-20.

5. Andrew DH (2008) Colouration of Hydroentangled Nonwoven Fabrics, Phd Theses, University of Leeds.

6. Zheng H, Seyam AM, Stiffler D (2003) The Impact of Input Energy on the Performance of Hydroentangled Nonwoven Fabrics, INJ, pp: 34-44.

7. Mehmet $Y$, Thomas $H$, Sabit A (2008) Influence of the fabric properties on fabric stiffness for the industrial fabrics. Tekstil ve Konfeksiyon 4: 263-267.

8. Smith K, Ogale AA, Maugans R, Walash LK, Patel RM (2003) Effect of bond roll pattern and temperature on the micro structure and properties of polyethylene nonwoven. Textile Res J 70: 845-853.

9. Kristina A, Eugenija S, Anastasija N (2010) The Relationship between Fabrics Bending Rigidity Parameters Defined by KES-F and FAST Equipment. Materials Science 16: 346-352.

10. Komori T, Itoh M (1997) Analysing the compressibility of a random fibre mass based on modified theory of fibre contact. Textile Res J 67: 204-210.

11. Bahari N, Hasani H, Zarrebini M, Hassanzadeh S (2015) Investigating the effects of material and process variables on the mechanical properties of lowdensity thermally bonded nonwoven produced from estabragh (milkweed) natural fibers. $\mathrm{J}$ Ind Text 46: 719-736.

12. Berrfelder $P$, Dorrestijn $M$, Roth $C$ (2013) Effect of fibre count and knit structure on intra and inter-yarn transport of liquid water. Text Res J 83: 1477-1488.

13. Hassan M, Qashqary K, Hassan HA, Shady E, Alansary M (2012) Influence of Sportswear Fabric Properties on the Health and Performance of Athletes. Fibres Text East Eur 20: 82-88. 
Citation: Cheema SM, Shah TH, Anand SC, Soin N (2018) Development and Characterisation of Nonwoven Fabrics for Apparel Applications. J Textile Sci Eng 8: 359. doi: 10.4172/2165-8064.1000359

14. Firgo H, Schustera KC, Suchomela F, Männera J, Burrowa T, et al. (2006) The Functional Properties of Tencel $^{\circledR}$ - A Current Update. Lenzinger Berichte 85: 22-30.

15. Miller B (2000) Critical Evaluation of Upward Wicking Tests. Int Nonwovens J Vol: 9.

16. Cannolly TJ, Parent LR (1993) Influence of specific energy on the properties of hydroentangled nonwoven fabrics. Tappi Journal 76: 135-141.

17. Ghassemieh E, Acar M, Versteeg K (2001) Improvement of the efficiency of energy transfer in the hydroentanglement process. Compos Sci Technology 62: $1681-1694$
18. Mao $N(2000)$ effect of fabric structure on the liquid transport characteristics of nonwoven wound dressing, PhD thesis, University of Leeds, UK, pp: 1-253.

19. www.paperdressvintage.co.uk/livintage-boutique/history.

20. Russell SJ (2007) Handbook of Nonwoven, Woodhead Publishing Limited (Istedn), 1: 286-287.

21. Shawver SE, Collier LW, Estey PW, Paul SC (1997) Elastic Breathable Barrier Fabric, US Patent 5: 695-849.

22. https://store.extension.iastate.edu/Product/pm1663d-pdf. 